\title{
« Faire du neuf avec du vieux »
}

Chrétien de Troyes relu par Raoul de Houdenc dans Méraugis de Portlesguez

Carine Giovénal

\section{(2) OpenEdition}

\section{Journals}

Édition électronique

URL : http://journals.openedition.org/aes/385

DOI : 10.4000/aes.385

ISSN : 2258-093X

Éditeur

Laboratoire LISAA

\section{Référence électronique}

Carine Giovénal, « "Faire du neuf avec du vieux » », Arts et Savoirs [En ligne], 3 | 2013, mis en ligne le 15 février 2012, consulté le 01 mai 2019. URL : http://journals.openedition.org/aes/385 ; DOI : 10.4000/ aes.385

Ce document a été généré automatiquement le 1 mai 2019.

Centre de recherche LISAA (Littératures SAvoirs et Arts) 


\title{
«Faire du neuf avec du vieux »
}

\author{
Chrétien de Troyes relu par Raoul de Houdenc dans Méraugis de \\ Portlesguez
}

Carine Giovénal

1 Nombreux sont les qualificatifs qui désignent Raoul de Houdenc, auteur du début du XIII siècle d'un roman arthurien et d'un récit allégorique : épigone de Chrétien de Troyes, continuateur ou encore disciple du maître champenois. Comme bien d'autres écrivains de cette période, il existe en référence à, voire dans l'ombre du maître de la matière arthurienne. Et de fait, la lecture de son Meraugis de Portlesguez, roman d'aventures du début du XIII ${ }^{e}$ siècle, entraine le lecteur sur le terrain connu des romans de Chrétien de Troyes, dont Raoul reprend bon nombre de situations et de motifs - au sens d'images ou d'actions inscrites dans la tradition littéraire arthurienne, que ce soit le tournoi initial désignant le plus preux et la plus belle, l'enamoratio, la quête en des lieux étranges et enchantés, l'enlèvement de la dame ou les retrouvailles finales des amants.

2 Mais Raoul ne se limite pas à retravailler les motifs mis à l'honneur par Chrétien; il modèle la matière bretonne dans une indéniable intention parodique. Entendons ici la parodie, non comme la dénonciation subversive d'une auctoritas que respectaient les auteurs médiévaux, mais plutôt comme " une habile contrefaçon où s'expérimente, sur le mode ludique, tout le talent de l'imitateur $»^{1}$. Concernant les emprunts de Raoul envers Chrétien, doit-on parler d'irrévérence, d'un simple jeu libérateur, ou d'un véritable moteur de rajeunissement littéraire? Nous tâcherons de répondre à ces questions en voyant d'abord comment Raoul de Houdenc déforme les motifs célèbres du roman arthurien dans une indéniable intention parodique. Notre étude s'attachera ensuite au personnage de Méraugis, jeune chevalier qui se démarque singulièrement de ses aînés littéraires par un comportement des plus burlesques. Enfin, nous verrons comment ces effets comiques permettent à l'auteur, au-delà de l'aspect divertissant, de prendre ses distances avec un modèle arthurien trop exploité et de proposer une nouvelle vision du chevalier. 


\section{Reprise ironique d'un espace-cadre connu}

En premier lieu, observons le tournoi initial. Les deux futurs amants, Méraugis et Lidoine, se rencontrent lors de joutes organisées par la dame de Landemore, joutes qui doivent désigner le chevalier le plus vaillant et la demoiselle la plus belle. On se souvient de l'épisode similaire du début d'Erec, alors que le héros éponyme, hébergé par le père d'Enide, écoute celui-ci lui expliquer les règles du tournoi :

[...] devant trestote la gent

Iert sor une perche d'argent

Uns espreviers molt biax assis

Ou de cinc mües ou de sis,

Le meillor q'an porra savoir.

Qui l'esprevier voldra avoir,

Avoir il covandra amie

Bele et saige sanz vilenie ;

S'il i a chevalier si os

Qui vuelle le pris et le los

De la plus bele desresnier,

S'amie fera l'esprevier

Devant toz a la perche prandre,

S'autres ne li ose desfandre.

Iceste costume maintienent,

Et por ce chascun an i vienent. ${ }^{2}$

Raoul de Houdenc reprend ce motif et l'intègre à Meraugis. Mais les règles qu'il donne au tournoi de Lindesores, auquel se rendent Méraugis et Lidoine, diffèrent sensiblement de celles qu'avait choisies Chrétien de Troyes :

Qui l'onor porra assentir'

De vaintre le tornoiement,

Si en portera quitement

Un cisne qui el pin sera.

E lors vos di qu'il baisera

La pucele de Landemore,

Qui n'est mie laide ne more.

Quant li cisnes sera donez,

Maintenant ert uns cors sonez

A la fontaine souz le pin.

Sus une lance de sapin

Sera uns esperviers muez,

Qui ja n'iert pris ne remuez

Devant la que cele le pregne

Qui par veüe lor apregne

Qu'ele soit plus bele que totes.

Se la robe ert perciee as coutes

Por tant que ce fust la plus bele,

N'i avra il ja damoisele

Qui ja l'emporte se li non.

Car il sera donez par non

A cele qui ert esleüe

Estre la plus bele a veüe.

Einsi fu lors li tornois pris. ${ }^{3}$ (v. 134-157)

On retrouve chez Raoul les mêmes motifs que chez Chrétien : l'épervier à remettre à la plus belle dame, et l'élection du meilleur chevalier. De surcroît, il reprend en filigrane 
l'épisode d'ouverture d'Erec, celui de la chasse au blanc cerf, dont le vainqueur gagnera le droit d'embrasser la plus belle. Mais Raoul de Houdenc n'a repris ces éléments que pour mieux les détourner. La perche d'argent d'Erec devient chez Méraugis une lance de sapin; à l'amie bele et sage sanz vilenie se substitue la pucele de Landemore, qui n'est mie laide ne more; enfin, le fait de remettre un cisne au vainqueur est ambigu: le cygne est blanc dehors, mais noir dedans, ce qui peut sous-entendre que celui qui remportera le tournoi ne sera peut-être pas le plus méritant... ${ }^{4}$ Ce qui sera exactement le cas! Raoul s'ingénie à démonter le motif traditionnel du tournoi rituel par une ironie subtile, en laissant entendre la raillerie sous la louange. On note aussi chez Meraugis la mention du cor sonez a la fontaine souz le pin; la fontaine est reprise d'Yvain, et le cor d'Erec. Mais chez Erec, le cor est sonné pour annoncer la victoire finale du héros et la diffusion générale de la joie. Or, chez Meraugis, on sonne le cor au début du roman, alors que les mésaventures du héros qui n'a pas même encore été présenté - ne font que commencer.

Raoul ne se contente pas de détourner les motifs ; il agit de même avec le schéma narratif. À la régularité de la conjointure d'Erec, qui à l'issue du tournoi sacre le couple idéal - Erec le meilleur chevalier, Enide la plus belle dame qui remporte sans conteste l'épervier -, le tournoi de Meraugis divise le prix à gagner, et par là même sa valeur : le meilleur chevalier remporte un cygne et un baiser de la dame de Landemore, la plus belle dame un épervier. Ainsi, comme le souligne Michelle Szkilnik dans son introduction à l'édition de Meraugis :

[...] alors que chez Chrétien, la vaillance d'un chevalier est proportionnelle à la beauté de son amie, idée qui est au fondement de l'idéologie courtoise, chez Raoul les deux prix sont séparés et sanctionnent deux valeurs présentées comme indépendantes. ${ }^{5}$

La molt bele conjointure d'Erec se trouve ici fortement mise à mal, tant dans les règles du tournoi, qui séparent ce qui devrait être conjoint, que dans le déroulement même de ce tournoi. Chez Chrétien, il est évident qu'Erec est le meilleur jouteur présent sur la lice :

Molt feisoient de lui grant los,

Petit, et grant, et gresle, et gros ;

Tuit prisent sa chevalerie

N'i a chevalier qui ne die :

« Dex, quel vasal, soz ciel n'a tel. ${ }^{6}$

Chez Raoul, le vainqueur ne l'est que par complaisance, et l'auteur n'en fait pas mystère :

Or vos ferai savoir par non

Qui ot le cisne e le besier.

- Qui l'ot? - Caulas, uns chevaliers

Mout hardis d'armes, l'emporta.

E il plus s'i acreanta,

Non pas por ce qu'il n'i eüst

Mellor de lui qui l'esleüst,

Mes la damoisele l'amoit

E il li tant qu'il en aloit

Par tot aventures querant.

Si l'em porterent trestuit garant

Por fere li son dit estable,

N'ele n'est pas si covoitable

Que nuls l'en vousist fere tort.

Tuit se tindrent a son acort

Qu'il ont sor li l'entente mis. (v. 286-301) ${ }^{7}$

Et Raoul de conclure que cette épreuve emblématique du roman arthurien est décidément vidée de son sens : 
Mes ce fu par le gré d'amis

Qu'il ot le cysne e le besier,

Car qui vouzist reson cerchier,

Mellor de lui trovast encor. (v. 302-305) ${ }^{8}$

On aura aussi relevé le commentaire peu charitable de l'auteur quant à la beauté de la dame de Landemore, pas si covoitable qu'on ait voulu se battre pour avoir l'honneur de son baiser. Et que dire de cette malicieuse allusion en parlant de la robe de la dame - encore inconnue - qui sera désignée comme la plus belle :

[...] Devant la que cele le pregne

Qui par veüe lor apregne

Qu'el soit la plus bele que totes.

Se la robe ert perciee as coutes

Por tant que ce fust la plus bele,

N'i avra il ja damoisele

Qui ja l'emporte se li non. (v. 147-153) ${ }^{9}$

Les familiers des romans arthuriens auront sans peine reconnu la mention de la robe usée d'Enide lors de sa première apparition devant Erec, li chainses viez / Que as costez estoit perciez $^{10}$. Raoul s'amuse ici à prendre le contre-pied de Chrétien: alors qu'on devine rapidement que c'est Lidoine qui va remporter le prix de la beauté, on ne peut que savourer l'ironie de ce parallèle: la riche suzeraine d'Escavalon ne va certes pas se présenter au tournoi avec une robe trouée!

L'enamoratio des héros de Houdenc est soumise au même traitement ironique que le tournoi. Lorsqu'Erec et Enide se rencontrent, Chrétien de Troyes décrit l'instant avec concision et sobriété :

Quant [Enide] le chevalier voit,

Que onques mes veü n'avoit,

Un petit arriere s'estut:

Por ce qu'ele ne le quenut,

Vergoigne en ot et si rogi.

Erec d'autre part s'esbahi

Quant an li si grant biauté vit. ${ }^{11}$

Nul besoin d'une longue description pour faire comprendre au lecteur le sentiment qui vient de naître, par ce seul échange de regards, entre les deux jeunes gens. L'enamoratio est immédiate, spontanée, et réciproque, ce qui la rend d'autant plus émouvante.

Dans Meraugis, le schéma amoureux initial rappelle plutôt celui du Chevalier à la Charrette : le jeune chevalier tombe éperdument amoureux d'une puissante suzeraine qui, dans les premiers temps, ne semble pas répondre à ses sentiments. L'enamoratio viendra avec l'échange du premier baiser qui révèle à la dame la vérité de son cœur: elle aime Méraugis. Mais cet instant intime est traité par l'auteur sur un ton ironique qui remet en cause la tradition méliorative de l'amour courtois. L'acte du baiser d'amour est dégradé par l'exagération comique de ses prétendues vertus, exagération à laquelle se livre complaisamment le narrateur :

[...] E savez qu'[e Meraugis] i em porta

E de quel mors il fu empliz?

Au mains fu il mout raempliz

Par ce besier de touz les biens

Si plesaument qu'il n'i faut riens

Que bons chevaliers doie avoir.

Par cest besier poez savoir

Quen itel proëce illumine

Que mout avroit aillors mecine. 
Mecine? Certes, ç'avroit mon!

L'en escoute poior sarmon

Mainte foiz. [...] (v. 1144-55) ${ }^{12}$

On aura relevé les termes hyperboliques tels quel mors, mout, touz, si, il n'i faut riens, itel, et aussi l'auto-dérision avec la mention du poior sarmon ${ }^{13}$ : Raoul se moque à la fois des métaphores courtoises excessives et du discours qu'il en fait, laissant entendre que le narrateur lui-même ne croit pas à ce qu'il raconte!

C'est de Cligès que s'inspire cette fois Raoul, précisément de l'enamoratio de Soredamor et Alexandre, parents du héros éponyme. Chrétien lui-même décrivait cette enamoratio avec un excès humoristique : dans un dialogue fictif entre le personnage et son moi, Amour est allégorisé à outrance, et des métaphores filées hyperboliques, héritées d'Ovide, s'enchaînent inlassablement sur les thèmes de la flèche d'amour qui pénètre par les yeux pour introduire un feu dévorant dans le cœur de sa victime :

$-[. .$.$] qu'[Amors] m'a navré si fort$

Que jusqu'au cuer m'a son dart trait

Met ne l'a pas a lui retrait.

Comant le t'a donc trait el cors

Quant la plaie ne pert de fors?

Ce me diras : savoir le vuel!

Comant le t'a il tret? Par l'uel.

Par l'uel ? Si ne le t'a crevé?

A l'uel ne m'a il rien grevé ;

Mes au cuer me grieve formant. [...]

De ce sai je bien reison randre :

Li ialz n'a soin de rien antandre

Ne rien ne puet feire a nul fuer ;

Mes c'est li mereors au cuer,

Et par ce mireor trespasse,

Si qu'il ne blesce ne ne quasse,

Le san don li cuers est espris.

Donc est li cuers el vantre mis

Aussi com la chandoile esprise

Est dedanz la lenterne mise.

Se la chandoile an departez,

Ja n'an istra nule clartez ;

Mes tant con la chandoile dure,

Ne est pas la lanterne oscure,

Et la flame qui dedanz luist,

Ne l'anpire ne ne li nuist [...] (v. 684-716) ${ }^{14}$

La forme du dialogue est présente également chez Meraugis, mais entre le narrateur et un narrataire virtuel, lequel relance l'effet comique par des questions et commentaires faussement naïfs. L'enamoratio des parents de Cligès, déjà présentée de manière ironique chez Chrétien, l'est de manière subversive avec Raoul.

Cependant, le baiser donné par Méraugis fait naître l'amour en Lidoine, un amour total et irrépressible que Raoul de Houdenc nous révèle en utilisant à contre-pied les métaphores ovidiennes vues ci-dessus :

- Lidoine vint trop doucement

Au chevalier. Avint einsi

Q'un poi d'amor de lui issi

Qui encontre Lidoine vint,

Si q'au point dou besier avint

Qu'il li lança au cuer dedens, 
N'onques ne li feri as denz

L'amor quant ele i fu lancie. (v. 1166-73) ${ }^{15}$

La mention des denz, incapables de stopper l'élan de passion, matérialise et dévalorise la naissance éthérée et la réciprocité parfaite du sentiment amoureux. Raoul poursuit en continuant de filer la métaphore. La naissance de la passion se réduit à une plaisante pêche à la ligne où Méraugis est le pêcheur, l'amour l'hameçon, et le cœur de Lidoine le poisson :

- Mes Dex, de qoi aaschie

L'amor qui dedenz aus vola?

- Ne sai, mes ses cuers l'engoula

Ausi com li poissons fet l'aim. [...] (v. 1174-77) ${ }^{16}$

Le verbe engoula réduit cette enamoratio à la trivialité d'un acte d'ingestion. Puis les rôles s'inversent: Méraugis devient à son tour poisson, et les yeux de Lidoine le filet de pêche qui l'attrape :

- [Lidoine] le feri des iex une foiz,

E amor se fiert en la roiz. [...]

L'en voit au cors

Que li oil peschent les amors

Que c'est la roiz as amanz prendre.

Par tant poez des iex apprendre,

Que c'est voirs. [...] (v. 1193-1201) ${ }^{17}$

Et le narrateur de conclure d'un même ton cette partie de pêche amoureuse qui s'est avérée, pour Lidoine, bien fructueuse :

$-[$...] Cele se cuidoit

Garder, mes il tendi devant.

- Et que prist il ? - Des amors tant

Q'uns autres s'en feïst a mains.

E lors quant ses batiax fu plains

Lidoine s'esmervelle [...]. (v. 1204-1209) ${ }^{18}$

Il semble que Raoul connaisse le Traité de l'amour courtois de son contemporain André Le Chapelain, qui utilise lui aussi la métaphore du pêcheur pour expliquer l'enamoratio :

[...] celui qui aime est pris dans les chaînes du désir et il souhaite prendre l'autre à son hameçon. De même en effet que le pêcheur adroit s'efforce de ferrer les poissons grâce à ses appâts et de les prendre à son hameçon crochu, de même celui qui est pris par l'amour s'ingénie à attirer l'autre par des manœuvres de séduction $[\ldots] .{ }^{19}$

Les jeux de mots sur «amour, aimer" (amor, amare) et « hameçon, crochet, pêcher à l'hameçon» (hamus, hamare) sont fréquents dans toute la littérature du Moyen Âge ${ }^{20}$. Raoul reprend ce thème bien connu et le grossit à outrance. Les situations familières aux lecteurs des romans arthuriens sont exagérées, déplacées, détournées de leur but traditionnel et prêtent à rire.

10 Après avoir repris et bestourné ${ }^{21}$ les schémas narratifs connus, Raoul de Houdenc va maintenant faire subir le même traitement comique au héros masculin de son roman, Méraugis de Portlesguez.

\section{Un surprenant chevalier}

11 Descendant en droite ligne des plus grands personnages arthuriens - le Tristan en prose fait de lui le fils du roi Marc -, le jeune Méraugis apparaît dans ce roman éponyme, à première vue, comme un héros traditionnel du monde arthurien. Chevalier mout alosez ${ }^{22}$ (v. 320), c'est un bachelier qui va s'aguerrir de tournoi en tournoi, à l'exemple d'Yvain. Il 
est un combattant émérite, ainsi que Lidoine a pu le constater lors du tournoi initial donné à Lindesores :

E cele [Lidoine] s'est contr'aus [Meraugis e Gorvain] levee,

Qui bel lor rendi lors salus,

Qu'el les ot le jor conneüz.

Que ce furent li dui por voir

Ax cox doner e recevoir

Qui miex le firent en l'estour

E qui plus li plorent le jor. (v. 358-364) ${ }^{23}$

Méraugis est aussi un chevalier parfaitement courtois : tombé amoureux de Lidoine en même temps que son compagnon Gorvain Cadruz, il a la finesse d'aimer la dame pour sa courtoisie, alors que Gorvain l'aime pour sa beauté ${ }^{24}$. Cette capacité à distinguer et apprécier la vraie valeur de Lidoine lui permet d'être désigné comme son prétendant officiel, quelques temps plus tard, à la cour du roi Arthur.

Cependant, lorsque Lidoine et Méraugis partent ensemble en quête de Gauvain - disparu de la cour depuis un an -, le comportement du chevalier va se révéler surprenant. Là où l'on attendait mesure et réflexion de la part d'un jeune homme si parfaitement courtois, Méraugis va au contraire se livrer à des actes plus qu'inattendus de mémoire de lecteur.

Une des épreuves qui dévoilent le caractère immature du héros est celle de l'écu, au commencement de la quête pour retrouver Gauvain. Une vieille demande à Méraugis de faire tomber un écu suspendu à un arbre ; cela fait, elle rendra le cheval qu'elle vient de dérober au nain chargé de guider le héros. Tout chevalier expérimenté, ou du moins doté de prudence, se serait renseigné sur les conséquences de la chute dudit bouclier. Mais Méraugis, pressé de bien faire, ne s'embarrasse pas de questions :

Lors s'eslesse e point d'escoeillie,

L'escu abat e ou repaire [...] (v.1495-96) ${ }^{25}$

Or, en abattant l'écu, Méraugis provoque les pleurs de deux demoiselles se trouvant à proximité. Comprenant d'instinct qu'un grand malheur menace, Lidoine se met à pleurer avec elles. Méraugis, en revanche, ne comprend rien à ces larmes et s'affole :

Quant li chevaliers voit s'amie

Qui pleure, a poi qu'il n'est desvez.

"Q'est-ce, fet il, por qoi plorez?»

E la pucele respondié :

«Sire, je plor car j'ai pitié

De cez dames qui tel doel font

E si sai bien qu'eles le font

Por la pitié de cel escu. [...] (v. 1531-38) ${ }^{26}$

La réaction de Méraugis est alors bien naïve : il pense corriger son erreur en remettant tout bonnement le bouclier en place :

- Comment, a il dont tel meffet?

Fet cil qui nul mal n'i pensa.

Nenil ! N'en plorent eles ja

Que c'est legier a amender»

Lors prent l'escu, sel vet porter

Arriere la ou il pendoit. (v. 1541-46) ${ }^{27}$

On remarque l'intervention du narrateur et la formule périphrastique pour désigner le chevalier : cil qui nul mal n'i pensa. Cette formule est appropriée pour décrire le caractère de Méraugis qui, effectivement, ne « pense » pas beaucoup à l'orée de ses aventures, et se laisse facilement manipuler et berner. Aux sarcasmes d'une troisième demoiselle, il répond, contrit : 
[...] Hui mes n'en soi ge rien.

Mes ge cuidai fere mout bien. (v. 1554-55) ${ }^{28}$

L'emploi du verbe cuidai, " croire à tort », souligne le manque de réflexion du héros. Plein de bonnes intentions, Méraugis est sans malice, mais pas sans réaction. Les moqueries de cette demoiselle, jointes aux pleurs incessants et aux refus d'explication des deux autres jeunes filles, l'échauffent brusquement, et il passe sa frustration sur le bouclier qu'il abat une seconde fois :

Li chevaliers fu corrouciez [...]

L'escu qui au fresne pendoit

Reprent as mains, sel gete loig [...] (v. 1596-1602) ${ }^{29}$

Avant de conclure tel un enfant boudeur :

Atant s'assiet et dit : « Par m'ame,

Ge ne demant se guerre non [...]» (v. 1623-24) $)^{30}$

Cette réaction puérile peut nous rappeler les aventures de Perceval, le premier nice inventé par Chrétien de Troyes. Mais Perceval avait été élevé loin du monde des chevaliers et, si ses réactions étaient inappropriées, elles étaient compréhensibles et excusables. Méraugis, en revanche, chevalier mout alosez ${ }^{31}$, n'est pas à la hauteur de sa réputation: imprudent, emporté, il accumule les maladresses sans discernement ni réflexion, donnant à ses aventures une tonalité inattendue et comique, voire parodique ${ }^{32}$.

Méraugis est un très bon guerrier. Il ne craint aucun adversaire et remporte sans difficulté tous ses duels. Pourtant, lorsque nous arrivons au milieu du roman, le narrateur le fait se sortir d'une situation périlleuse d'une façon plus qu'originale pour un chevalier arthurien.

Toujours en quête de Gauvain, Méraugis et Lidoine sont parvenus à la Cité sans Nom. Là, Méraugis s'embarque pour une île où l'attend une épreuve, tandis que son amie reste sur la rive. Arrivé sur l'île, Méraugis doit affronter rituellement le chevalier gardien des lieux ; s'il parvient à le vaincre, l'île et le château lui reviendront, ainsi que la belle dame qui en est la maîtresse. Raoul utilise ici le thème millénaire du combat masculin ayant pour récompense une femme, figure de souveraineté, et la terre dont elle est l'incarnation, toutes deux devant légitimement revenir à l'homme le plus fort. Ce motif était déjà celui exploité par Chrétien de Troyes pour Yvain ou Le Chevalier au Lion ${ }^{33}$. Mais là où les chevaliers de Chrétien s'affrontent vaillamment pour conquérir les proies offertes à leur convoitise, les chevaliers de Raoul ont une réaction strictement inverse. En effet, le chevalier gardien de l'île s'avère être Gauvain. Loin d'être épris de la dame qui s'est offerte à lui, il la présente comme une mante religieuse qui le retient de force, comme l'avait été avant lui le précédent gardien des lieux :

[...] Une dame est, deci entor

La plus bele c'onques veïs.

Cele cité e cist païs

Est touz siens, que jadis avint

Q'uns chevaliers mout hardiz vint

S'a li. Si la requist d'amors

A euls commença en decors

Qu'ele l'ama, si fu s'espouse.

[...] S'ele nel commandast

E plus, que ja mes ne passast

Nus chevaliers parmi sa terre

Qui ne venist ça por conquerre

Le pris contre son champion.

[...] Que ge ving ça 


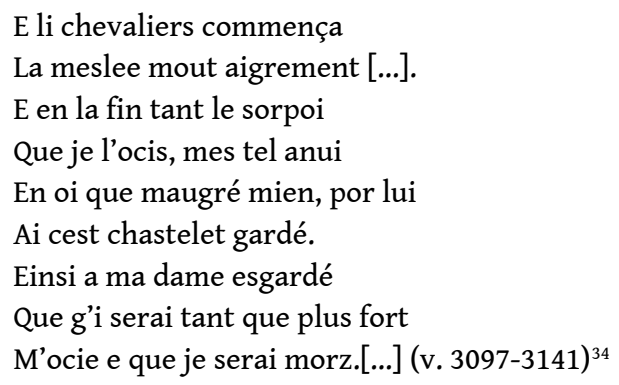

Et loin de revendiquer avec force son rôle de gardien-amant et de vouloir le conserver à tout prix, le neveu d'Arthur ne montre que résignation et désespoir à subir une fonction dont il ne veut pas :

[...] Or esgarde donques einsi :

Se tu me vains ou ge t'oci,

Comment qu'il aut, c'est li usages,

Li uns en remaindra en gages [...].

Einsi par force te covient

Combatre a moi. Ge n'i voi plus [...]. » (v. 3142-48) ${ }^{35}$

Même réaction de la part de Méraugis : le pris offert ne l'intéresse plus :

- De ce n'ai ge pas grant envie

Dit Meraugis, je n'en serai

Chastelains, non! Car je ne sai

Chastel qui tant face a haïr. [...] (v. 3153-56) (v6 $^{36}$

Cessant soudain d'être le jeune irréfléchi qu'il avait été jusque-là, Méraugis imagine alors une solution ingénieuse afin que lui et Gauvain s'échappent de ce piège. Puisque la dame et ses sujets sur la berge veulent un combat et une mise à mort, Gauvain et lui vont faire semblant de s'affronter ; Méraugis feindra d'être terrassé, et Gauvain ira jusqu'à montrer bien haut son heaume por miex decevoir la gent (v. 3217) ${ }^{37}$. Là où traditionnellement la force prime, ici, la ruse s'impose.

17 Mais c'est le stratagème de Méraugis pour quitter l'île qui s'avère inédit et franchement comique : un bateau vient régulièrement approvisionner les insulaires, et n'aborde que si la dame elle-même se montre sur la rive. Le narrateur souligne alors -malicieusement l'intense réflexion à laquelle se livre son héros pour résoudre le problème :

Meraugis s'apensa a point.

E quant il ot pensé, si fist. (v. 3297-98) ${ }^{38}$

Méraugis va donc poursuivre sa mise en scène à un stade très supérieur : le chevalier se travestit en femme! Pour souligner l'aspect parodique de cet épisode, une variante du manuscrit WB l'introduit par une apostrophe employée dans les récits épiques: Or escoutez si :

[...] - Par foi, il prist

Trestote la robe a la dame,

E lors dou tot come une fame

Se vest et lace e empopine.

Plus acesmez q'une popine,

Descent aval de cest chastel,

S'espee desoz son mantel. [...] (v. 3299-3305) ${ }^{39}$

Et le narrateur d'insister complaisamment sur l'aspect seyant de ces vêtements féminins sur le jeune et beau chevalier :

- [...] Que vos diroie? Au havre vint

Einsi vestuz. Mout li avint

Car il estoit bien fes et genz. (v. 3306-08) ${ }^{40}$ 
Et cette ruse se révèle efficace ! Abusés par la gracieuse dame qui leur fait signe depuis la rive, les marins abordent sans méfiance. Soulignons ici une autre remarque savoureuse du narrateur, lorsque Méraugis, toujours déguisé, saute dans le bateau, et que le poids de la « dame » manque le faire chavirer :

[...] E Meraugis

Que devant ot son conseil pris

Saut en la nef de plain eslés.

Si samble que totes les és

De la nef froissent e estendent. (v. 3316-20) ${ }^{41}$

La première partie du roman illustre un décalage entre un chevalier naïf et un monde extérieur sans pitié. Au milieu du roman où se situe l'aventure sur l'Ile sans Nom, ce schéma s'inverse : désormais, c'est Méraugis qui fera preuve d'un esprit acéré, n'hésitant pas à bouleverser les conventions romanesques pour parvenir à ses fins. Par ses initiatives inattendues, décalées et efficaces, le chevalier bestourne ${ }^{42}$ l'image traditionnelle du chevalier arthurien qui ne jure que par la force physique. La vision proposée se fait «burlesque ", la représentation du personnage et son comportement prennent un aspect ridicule qui contraste avec les portraits canoniques prévalant dans les romans antérieurs. L'auteur propose des solutions comiques nouvelles qui, en démontant les schémas classiques, donnent une vision neuve de l'homme et du chevalier.

\section{Raoul de Houdenc et l'effet comique}

Pourquoi Raoul choisit-il d'adapter la matière arthurienne, mise à l'honneur au siècle précédent par Chrétien, avec une tonalité comique?

À de nombreuses reprises, Raoul parodie Chrétien de Troyes, parodie dans le sens de jeu littéraire de transformations de modèles célèbres et respectés. Ses bestournements des schémas et personnages canoniques révèlent l'ingéniosité, l'engin ${ }^{43}$ de l'auteur face à une auctoritas à la fois honorée et remise en question, et lui permettent de tracer son message et sa propre voie littéraire.

Si l'épisode de la Cité sans Nom a des réminiscences d'Yvain, il est aussi une réécriture subversive de la Joie de la Cour d'Erec ${ }^{44}$. Comparons les deux schémas: dans Erec, Mabonagrain est retenu prisonnier depuis sept ans dans un verger par son amie, à qui il a promis de ne jamais s'éloigner à moins d'être vaincu. Dans Méraugis, une dame s'est retirée avec son mari sur une île avec interdiction à quiconque d'y aborder, et obligation à tout chevalier de passage d'y être conduit de force pour affronter son époux ; c'est un combat à mort, et le vainqueur reste prisonnier de l'île dans l'attente d'un adversaire qui prendra peut-être sa place. Les deux schémas sont régis par la toute-puissante coutume.

2 Michelle Szkilnik note que dès l'énoncé des faits, Raoul laisse entendre « une critique de la coutume et des pressions absurdes qu'elle fait peser sur les chevaliers. Cette mise en cause de la coutume se discernait déjà dans la façon désinvolte dont Raoul avait repris l'épisode de l'épervier ${ }^{45}$. En effet, si dans Erec, le héros éponyme met fin à la coutume en surpassant Mabonagrain, répandant par cette victoire la Joie autour de lui, dans Méraugis, Gauvain, qui a tué l'époux de la dame, ne met pas un terme à la coutume. Celle-ci est condamnée à se répéter indéfiniment, au rythme des chevaliers qui auront la malchance de passer par la Cité sans Nom. Aucun bénéfice ne peut être retiré de cette épreuve rituelle qui semble exister davantage pour avilir les combattants que pour accroitre leur prestige. L'alternative proposée est claire : 
[...] Se tu me vains ou ge t'oci,

Comment qu'il aut, c'est li usages,

Li uns en remaindra en gages [...]. (v. 3143-46) ${ }^{46}$

Réduits au statut de gages, de monnaie d'échange indifférenciée, les chevaliers en perdent leur identité. Ainsi Gauvain, "soleil de la chevalerie ", est devenu mélancolique et suicidaire :

[...] Ci a male vie,

Mout aim ma mort. Se ge pooie

Sauver ton ${ }^{47}$ cors, ge m'ocirroie

Tot orendroit sanz plus atendre. [...] (v. 3185-88) ${ }^{48}$

Le même schéma se met en place dans un épisode ultérieur, avec une carole magique qui happe Méraugis et lui fait perdre la mémoire, ne laissant vivre en lui que le désir irrépressible de danser ${ }^{49}$. Lancelot subit le même enchantement dans le Lancelot en prose $e^{50}$. Toutefois, Lancelot met fin à la magie de la parole, alors que Méraugis ne s'en échappe que par accident, quand un autre chevalier vient prendre sa place. À nouveau, les chevaliers ne sont que des objets interchangeables destinés à faire perdurer un topos narratif.

Cette réécriture de la Joie d'Erec est aussi pour Raoul l'occasion de reprendre un épisode de la Queste del Saint Graal, épisode résolument mystique : celui du château des Pucelles. Galaad, le Bon chevalier, se présente pour abolir la mauvaise coutume qui régit ce lieu : «Ce est li chastiaus maleoiz [...] len i fet honte a toz cels qui i trespassent. " ${ }^{51}$ Ayant vaincu les sept frères gardiens des lieux, Galaad libère les jeunes filles qui y étaient retenues. Commentée par l'ermite, l'aventure prend un sens religieux : les jeunes filles représentent les bonnes âmes retenues en enfer et délivrées par le Christ, les sept frères sont les sept péchés capitaux. Pour les lecteurs qui connaissent la Queste, on ne peut que savourer l'inversion à laquelle se livre Raoul, en faisant du château des Pucelles une île dominée par une femme castratrice qui exerce tout son pouvoir sur des chevaliers soumis. Outre l'inversion comique des sexes des acteurs, on relève la comparaison hautement subversive de Galaad à Méraugis, du saint chevalier avatar du Christ au jeune homme rusé qui se travestit en femme pour se sortir d'affaire!

En refusant d'abolir les mauvaises coutumes qui parsèment le monde arthurien, Méraugis se démarque de ses prédécesseurs littéraires. Si ce comportement inattendu amuse le lecteur, il révèle également une facette nouvelle du chevalier : celui d'un être qui agit en tant qu'individu qui poursuit une quête personnelle, là où ses aînés étaient engagés au service de la communauté. Les mauvaises coutumes que croise Méraugis ne font pas partie de ses priorités qui sont de retrouver Gauvain, puis Lidoine ; c'est pourquoi il ne s'attarde pas à les combattre. Rédigé dans le premier tiers du XIII ${ }^{\mathrm{e}}$ siècle, Meraugis de Portlesguez met en avant une figure d'homme nouveau en accord avec son temps : c'est en effet l'époque où l'individu commence à se distinguer du collectif et où l'être humain commence à aspirer à une reconnaissance personnelle, idées nouvelles encouragées par la puissance montante de la bourgeoisie et des valeurs croissantes de l'argent et de l'enrichissement personnel. Méraugis n'est pas au service de la Table Ronde, il ne combat pas pour Arthur ni pour sa communauté ; il est sans roi, sans lignage, sans terre. Ses seuls points d'ancrage sont son ami Gorvain au début du roman, son amie Lidoine par la suite, deux êtres qu'il a choisis et qu'il place avant le service du collectif. Une des raisons de son comportement irréfléchi et désinvolte se trouve dans cet élan individualiste qui le pousse à ne se préoccuper que de ses propres intérêts, rejetant le modèle du chevalier au service 
de l'autre mis à l'honneur au siècle précédent dans les romans de Chrétien. Méraugis ne sert que lui-même.

$\mathrm{Au} \mathrm{XIII} \mathrm{siècle,} \mathrm{le} \mathrm{roman} \mathrm{arthurien} \mathrm{en} \mathrm{vers} \mathrm{s'épuise.} \mathrm{La} \mathrm{matière} \mathrm{arthurienne,} \mathrm{surexploitée}$ depuis un siècle, cherche à se renouveler en explorant de nouvelles directions, que ce soit dans la violence extrême - L'Âtre périlleux ${ }^{52}$-, en détruisant littéralement les chevaliers fondateurs pour leur donner une nouvelle vie littéraire, ou en plaçant ces mêmes chevaliers dans des situations inédites, comiques, voire burlesques - Les Merveilles de Rigomer $^{53}$. Cette même matière arthurienne abandonnera bientôt le genre versifié pour trouver un élan nouveau avec les grands cycles des romans en prose, centrés autour de l'image mystique du Graal.

Dans l'intervalle, Raoul de Houdenc crée des configurations nouvelles, en faisant éclater les schémas connus pour mieux les reconstruire sur le mode ironique et comique. Cependant, son talent pour faire "du neuf avec du vieux" ne se limite pas à une recherche parodique et esthétique : il ancre son roman dans les préoccupations de son temps en faisant de son chevalier un homme qui cherche à exister par et pour lui-même, et non comme maillon indifférencié d'une communauté. L'aspect comique des aventures de Méraugis prend ainsi un sens nouveau: celui d'une quête qui, individuelle chez Chrétien de Troyes, se fait, un siècle plus tard, individualiste chez Raoul de Houdenc.

\section{NOTES}

1. Citation d'Elisabeth Gaucher, avant-propos de La Tentation du parodique dans la littérature médiévale, Cahiers de recherches médiévales, $\mathrm{n}^{\circ}$ 15, Champion, Paris, 2008.

2. Chrétien de Troyes, Erec et Enide, Paris, Champion : Les Classiques français du Moyen Âge, 1981, v. 565-580 : «Devant toute l'assistance, / il y aura sur une perche d'argent / un épervier assis fort joliment, / de cinq mues ou de six, / le meilleur qui se puisse trouver. / À celui qui voudra obtenir l'épervier, / il lui faudra avoir une amie / belle et sage, sans vilenie. / S'il se trouve chevalier assez hardi / pour oser revendiquer pour son amie / le prix et l'honneur de la plus belle, / il fera prendre l'épervier / par elle sur la perche aux yeux de tous, / à moins que quelqu'un n'ait l'audace de le lui défendre. / Pour maintenir cette coutume, / tous sont chaque année au rendezvous. " (Traduction tirée de Chrétien de Troyes, Erec et Enide, Paris, Lettres Gothiques, 1992, p. 69).

3. Raoul de Houdenc, Meraugis de Portlesguez, roman arthurien du XIII siècle, publié d'après le manuscrit de la Bibliothèque du Vatican, Édition bilingue, publication, traduction, présentation et notes par Michelle Szkilnik, Paris, Champion Classiques, 2004 : «Celui qui aurait l'honneur de remporter le tournoi gagnerait sans contestation un cygne qui serait juché sur un pin. Puis, je vous l'affirme, il embrasserait la jeune fille de Landemore qui n'était ni laide ni noiraude. Le cygne décerné, on sonnerait aussitôt d'un cor près de la fontaine sous le pin. Sur une pique de sapin serait perché un épervier déjà mué. Personne ne le prendrait ni ne le déplacerait avant que ne s'en saisisse la jeune fille qui, aux yeux du public, serait la plus belle de toutes. Pourvu qu'elle fût la plus belle, elle pourrait même porter une robe trouée aux coudes, aucune autre demoiselle qu'elle n'emporterait l'oiseau, car il reviendrait sans conteste à celle qui serait clairement 
désignée comme la plus belle. Ainsi furent établies les conditions du tournoi. » (Traduction de Michèle Szkilnik, édition citée).

4. On retrouve ce motif du cisne, oiseau ambigu, dans le roman contemporain La Queste del Saint Graal: «Li cisnes est blans par defors et noirs par dedenz, ce est li ypocrites, qui est jaunes e pales, e semble bien, a ce qui defors en apert, que ce soit serjanz Jhesuchrist; mes il est par dedenz si noirs e si horribles d'ordures et de pechiez qu'il engigne trop malement le monde ", p. 185-86, Honoré Champion Classiques, publié par Albert Pauphilet, Paris, 2003.

5. P. 25 de l'édition de Méraugis.

6. Erec et Enide, op. cit., v. 1245-1249: « Tous le comblaient d'éloges, / grands et petits, menus et gros./ Tous admirent ses qualités chevaleresques. Pas un chevalier qui ne dise : / «Mon Dieu, quel vassal! Sous le ciel, il n'a pas son égal. » »

7. « À présent je vais bien sûr vous dire qui emporta la cygne et le baiser. - Qui ? - C'est Caulas, un valeureux chevalier, qui l'emporta et qui accepta ce prix avec le plus d'empressement. Il y avait certes des chevaliers meilleurs que lui, à bien choisir, mais la demoiselle l'aimait et lui aimait tant la demoiselle que pour elle il allait partout en quête d'aventures. Tout le monde se porta garant de sa valeur afin de confirmer l'inclination déclarée de la dame. Celle-ci du reste n'était pas si désirable qu'on voulût la disputer à Caulas. Tous se rangèrent à l'avis de la dame. »

8. « Mais c'est grâce à la complaisance de ses amis qu'il emporta le cygne et le baiser car en toute justice, on aurait trouvé meilleur que lui ».

9. «Personne ne le prendrait ni ne le déplacerait avant que ne s'en saisisse la jeune fille qui, aux yeux du public, serait la plus belle de toutes. Pourvu qu'elle fût la plus belle, elle pourrait même porter une robe trouée aux coudes, aucune autre demoiselle qu'elle n'emporterait l'oiseau [...]». 10. Erec et Enide, op. cit., v. 407-08: «Une vieille chemise percée aux coudes ».

11. Ibid., v. 443-449: «Quand Enide vit le chevalier, / qu'elle n'avait encore jamais vu, / elle se tint un peu en retrait : / ne le connaissant pas, / elle manifesta de la timidité et rougit. / Quant à Erec, il fut tout ébloui / par le spectacle d'une si grande beauté. »

12. «[...] Et savez-vous ce qu'il en retira et de quelles vertus il se trouva pénétré ? Au bas mot, de la manière la plus agréable, ce baiser le dota de toutes les qualités requises chez un bon chevalier. Vous le devinez, un baiser capable de rehausser ainsi l'éclat de la valeur chevaleresque pourrait avoir d'autres vertus médicinales! - Des vertus médicinales? - Eh oui, bien sûr! On entend souvent de plus sots discours [...]. »

13. Plus sots discours.

14. Chrétien de Troyes, Cligès, Les Classiques Français du Moyen Âge, Champion, Paris, $1982:$ « [... ] Amour m'a si profondément blessé qu'il m'a tiré sa flèche en plein cœur et il ne l'a pas encore retirée. -Comment te l'a-t-il donc fichée dans le corps quand on ne voit aucune plaie au-dehors? Tu me le diras, je veux le savoir ! Par où te l'a-t-il tirée ? - Par l'œil. -Par l'œil ? Et il ne te l'a pas crevé ? - À l'œil il ne m'a fait aucun mal, mais c'est au cœur que j'ai très mal. [...] C'est facile à expliquer. L'œil ne cherche pas à comprendre, et il est incapable de faire quoi que ce soit; mais c'est le miroir du cœur, et par ce miroir passe, sans l'abîmer ni le briser, le feu qui brûle le cœur. Le cœur n'est-il pas dans la poitrine comme la chandelle allumée qu'on met dans la lanterne ? Si vous enlevez la chandelle, aucune clarté n'en sortira ; mais tant que la chandelle dure, la lanterne n'est pas obscure et la flamme luit à travers sans l'abîmer ni lui causer de dommage. » (Cligès, de Chrétien de Troyes, traduction de Michel Rousse, Garnier Flammarion, Paris, 2006).

15. «Lidoine s'approcha doucement du chevalier. Or un peu d'amour s'échappa de lui et reflua vers Lidoine, de sorte qu'au moment du baiser Méraugis le lui lança au fond du cœur. Les dents ne parvinrent pas à arrêter l'élan de l'amour.»

16. «- Mais, par Dieu, avec quoi était appâté l'amour qui se ficha en eux ? - Je ne sais pas, mais le cœur de Lidoine l'avala comme le poisson avale l'hameçon. » 
17. «[...] Lidoine lui jeta un regard. L'amour tomba dans le filet. [...] On voit que dans le corps ce sont les yeux qui pêchent l'amour. C'est le filet aux amoureux. Voilà ce que vous pouvez apprendre sur les yeux, c'est la vérité [...]. »

18. "Lidoine se croyait en sécurité, mais son cœur tendit ses filets. Et qu'attrapa-t-il ? Une telle quantité d'amour qu'un autre se serait satisfait de moins. Et quand sa barque fut pleine, Lidoine [fut] stupéfaite [...].»

19. André Le Chapelain, Traité de l'amour courtois, Introduction, traduction et notes par Claude Buridant, Klincksieck, Paris, 1974, p. 49-50.

20. Déjà Isidore de Séville dans ses Étymologies faisait dériver ami de hamus, signifiant alors « lien d'affection » : les amis tiennent donc leur nom de hamus parce qu'ils sont attachés l'un à l'autre (Isidore de Séville, Étymologies, X, L, 5, texte établi, trad. et annoté par Jean-Yves Guillaumin et Pierre Monat, Traduction de : Etymologiarum, Besançon : Presses universitaires de Franche-Comté, 2004).

21. «Inversé ».

22. "Chevalier très renommé ".

23. " [Lidoine] se leva pour recevoir [Méraugis et Gorvain] et leur rendit gracieusement leur salut. Tout au long de la journée, elle les avait distingués car en vérité, c'étaient les deux chevaliers qui s'étaient le mieux illustrés durant la bataille, tant pour asséner les coups que pour les parer. Aussi les avait-elle regardés avec une complaisance particulière. »

24. Les auteurs du début $d u X^{X} I^{e}$ siècle sont nombreux à s'interroger sur l'opposition entre semblance et senefiance, notamment dans La Queste del Saint Graal, op. cit.

25. " Il s'élança alors et chargeant à toute vitesse, fit tomber le bouclier. »

26. "Quand le chevalier vit son amie pleurer, il en perdit presque la tête. "Que se passe-t-il ? demanda-t-il. Pourquoi pleurez-vous? - Seigneur, répondit la jeune fille, je pleure par compassion pour ces dames qui se désolent si profondément. Et je sais bien qu'elles le font par contrariété pour le bouclier. [...]»

27. «- Quoi ? Est-ce si terrible? reprit Méraugis qui ne voyait là nul mal. Mais non! Qu'elles cessent de pleurer, ce n'est pas difficile à réparer. » Il prit alors le bouclier et le rapporta là où il était accroché. »

28. [...] « Je ne savais pas de quoi il retournait. Je croyais bien faire. »

29. «Le chevalier fut agacé [...]. Il saisit le bouclier qui pendait aux branches du frêne, le jeta au loin [...]. »

30. «Il s'assit et ajouta : « Ma foi, tout ce que je veux, c'est la bataille [...]. » »

31. "Chevalier très renommé ».

32. Aux p.17-18 de son introduction de Méraugis de Portlesguez, Michelle Szkilnik rappelle un épisode similaire ayant lieu dans le Lancelot en prose : «Pour satisfaire les exigences d'une vieille qui maltraitait un nain, Yvain abat un écu blanc goté a noir, pendu près des pavillons, à la grande détresse de douze demoiselles. [...] Malheureusement, l'expérience d'Yvain n'est d'aucun secours à Méraugis qui se montre encore plus maladroit que son modèle. Yvain, lui, ne jetait pas deux fois l'écu à terre ! ", Lancelot en prose, éd. A. Micha, t. 4, Genève, Droz, 1979, p. 264-327.

33. Vainqueur d'Escanor, gardien de la fontaine magique, Yvain prend sa place et épouse sa veuve, Laudine de Landuc. (Yvain ou Le Chevalier au lion, Champion, Paris, 1997, v. 800 et sq.)

34. «[...] Il y a là une dame, la plus belle que tu aies jamais vue. Cette ville et cette contrée lui appartiennent. Jadis, un chevalier très vaillant vint la voir et la pressa de l'épouser. Au bout du compte, elle tomba amoureuse de lui et l'épousa. [...] Et en plus, elle commanda que tout chevalier qui passait par sa terre soit amené ici pour se mesurer à son champion. [...] Je passai dans l'île et le chevalier engagea un rude combat contre moi [...]. Et finalement je fus plus fort que lui et le tuai. Mais les conséquences en furent malheureuses car je suis devenu à sa place et contre mon gré le gardien de ce château. Ma dame a décidé que j'y resterai jusqu'à ma mort : jusqu'à ce qu'un chevalier plus fort que moi me tue. [...]» 
35. «Considère donc l'alternative: ou tu me vaincs ou je te tue. Quoi qu'il advienne, c'est la coutume, l'un de nous restera en otage jusqu'à l'arrivée de plus fort que lui. [...] Par conséquent tu es forcé de te battre contre moi. Je ne vois pas d'autre issue. [...]»

36. «- Cela ne me tente guère, répliqua Méraugis, je n'en serai jamais le maître, non! Car je ne connais pas de château plus détestable. [...]»

37. «Pour mieux tromper les gens ».

38. « Après avoir réfléchi mûrement à la situation, Méraugis passa à l'action. »

39. «[...] Eh bien, il prit tous les vêtements de la dame, puis il s'habilla, se laça et se pomponna exactement comme une femme. Mieux attifée qu'une poupée, il descendit du château, l'épée dissimulée sous son manteau. [...]»

40. «[...] Que vous dirai-je ? Il alla au port ainsi vêtu. Sa tenue lui allait très bien, car il était gracieux et beau. »

41. «[...] Méraugis qui avait arrêté son plan auparavant, sauta sur le bateau de tout son poids, faisant grincer et craquer de manière inquiétante toutes les planches du navire. »

42. « inverse».

43. "L'ingéniosité ».

44. Erec et Enide, op. cit., v. 5371 et $s q$.

45. Michelle Szkilnik, «Méraugis et la Joie de la Cité ", La Tentation du parodique dans la littérature médiévale, sous la direction d'Elisabeth Gaucher, Cahiers de Recherches Médiévales, $\mathrm{n}^{\circ} 15$, Champion, Paris, 2008, note 10 p. 116.

46. «Ou tu me vaincs ou je te tue. Quoi qu'il advienne, c'est la coutume, l'un de nous restera en otage jusqu'à l'arrivée de plus fort que lui [...]. »

47. Il s'agit du corps de Méraugis.

48. «[...] Quel malheur ! J'attends la mort avec impatience. Si je pouvais te sauver, je me tuerais aussitôt sans plus attendre [...]. »

49. Méraugis, v. 3623 et sq.

50. Lancelot en prose, t. 4, éd. Alexandre Micha, Genève, Droz, 1979, p. 234-35.

51. La Queste del saint Graal, éd. Albert Pauphilet, Paris, Champion, rééd. 1978, p. 47.

52. L'Âtre périlleux, roman de la Table ronde, éd. par Brian Woledge, Paris : H. Champion, 1936.

53. Les Merveilles de Rigomer, Saggi su romanzo del XIII secolo : Jaufré, Merveilles de Rigomer, Joufroi de Poitiers, Wistasse le Moine, Sir Orfeo, Lai du Trot,/ Margherita Lecco, Alessandria, Ed. dell'Orso, 2003.

\section{INDEX}

Mots-clés : comique, ironie, Troyes (Chrétien de), effet comique

\section{AUTEUR}

\section{CARINE GIOVÉNAL}

Université d'Aix-en-Provence 